\title{
Comparison of the T2-star Values of Placentas Obtained from Pre- eclamptic Patients with Those of a Control Group: an Ex-vivo Magnetic Resonance Imaging Study
}

\author{
Nursel Yurttutan1, Murat Bakacak², Betül Kızıldağ1 \\ ${ }^{1}$ Department of Radiology, Kahramanmaraş Sütçü İmam University School of Medicine, Kahramanmaraş, Turkey \\ ${ }^{2}$ Department of Obstetrics and Gynecology, Kahramanmaraş Sütçü İmam University School of Medicine, Kahramanmaraş, Turkey
}

Background: Endotel dysfunction, vasoconstriction, and oxidative stress are described in the pathophysiology of pre-eclampsia, but its aetiology has not been revealed clearly.

Aims: To examine whether there is a difference between the placentas of pre-eclamptic pregnant women and those of a control group in terms of their T2 star values.

Study Design: Case-control study.

Methods: Twenty patients diagnosed with pre-eclampsia and 22 healthy controls were included in this study. The placentas obtained after births performed via Caesarean section were taken into the magnetic resonance imaging area in plastic bags within the first postnatal hour, and imaging was performed via modified DIXON-Quant sequence. Average values were obtained by performing T2 star measurements from four localisations on the placentas.

Results: T2 star values measured in the placentas of the control group were found to be significantly lower than those in the pre-eclampsia group $(\mathrm{p}<0.01)$. While the mean T2 star value in the pre-eclamptic group was found to be $37.48 \mathrm{~ms}$ (standard deviation \pm 11.3 ), this value was 28.74 (standard deviation \pm 8.08 ) in the control group. The cut-off value for the T2 star value, maximising the accuracy of diagnosis, was $28.59 \mathrm{~ms}$ (area under curve: $0.741 ; 95 \%$ confidence interval: $0.592-0.890)$; sensitivity and specificity were $70 \%$ and $63.6 \%$, respectively.

Conclusion: This study, the T2 star value, which is an indicator of iron amount, was found to be significantly lower in the control group than in the pre-eclampsia group. This may be related to the reduction in blood flow to the placenta due to endothelial dysfunction and vasoconstriction, which are important in pre-eclampsia pathophysiology.

Keywords: Ex vivo, magnetic resonance imaging, placenta, pre-eclampsia, T2 star
Pre-eclampsia is one of the leading causes of maternal and perinatal mortality. Various factors, such as endothelial dysfunction, vasoconstriction, and oxidative stress, are described in the pathophysiology of pre-eclampsia. Although the aetiology has not been fully clarified, vascular dysfunction resulting in poor placentation is considered to be the main cause $(1,2)$.
Iron, which is present in the composition of many body molecules, such as cytochrome, haemoglobin $(\mathrm{Hb})$, and myoglobin, is stored as ferritin after it is absorbed by the mucosa of the small intestine. Fundamentally, iron is stored in the liver parenchyma and reticulo-endothelial system (3).

\footnotetext{
Address for Correspondence: Dr. Nursel Yurttutan, Department of Radiology, Kahramanmaraş Sütçü İmam University School of Medicine, Kahramanmaraş, Turkey Phone: +905300411141 e-mail: raddrnursel@gmail.com Received:25 October $2016 \quad$ Accepted: 11 March 2017 •DOI: 10.4274/balkanmedj.2016.1472

Available at www.balkanmedicaljournal.org

Cite this article as:

Yurttutan N, Bakacak M, Kızıldağ B. Comparison of the T2-star Values of Placentas Obtained from Pre-eclamptic Patients with Those of a Control Group: an Ex-vivo Magnetic Resonance Imaging Study. Balkan Med J 2017;34:412-6

${ }^{\circ}$ Copyright 2017 by Trakya University Faculty of Medicine / The Balkan Medical Journal published by Galenos Publishing House.
} 
Magnetic resonance imaging (MRI) is the best non-invasive imaging method to reveal iron loading (4). Iron ions that accumulate in tissue can cause local distortion in the relaxation of spins and the body's magnetic field due to their superparamagnetic features and thus cause T1 (longitudinal relaxation time), T2 (transverse relaxation time), and, especially, $\mathrm{T} 2$ star $(\mathrm{T} 2 *)$ (transverse relaxation time affected by magnetic field in homogeneity) shortening. This effect causes signal loss in the affected organ in proportion with the amount of iron stored $(5,6)$. T2*-weighted images have the greatest sensitivity in terms of detecting iron (7). There are many reasons for iron loading in the body. The aim of this prospective study was to examine whether there is a difference between the placentas of pre-eclamptic pregnant women and those of a control group in terms of $\mathrm{T} 2 *$ values, which are a quantitative indicator of the amount of iron and are obtained via MRI.

\section{MATERIALS AND METHODS}

\section{Design}

Informed consents were obtained from all patients and approval for the study was granted by the Ethics Committee of Sütçü İmam University of Medical Sciences (No: 06, Date: 14 December 2015). The study included 20 pre-eclamptic placentas obtained from Caesarean section delivery and 22 healthy placentas as a control group. The mean maternal age was $28.3 \pm 5.131$ years in the pre-eclamptic group and $31 \pm 5.033$ years in the control group. Placentas with gross placental haematoma and gross calcification, placentas with multiple pregnancies, and placentas with foetal major chromosomalstructural abnormalities were excluded. Demographic features and detailed obstetric and medical data were collected from the hospital information system and prenatal care records. The

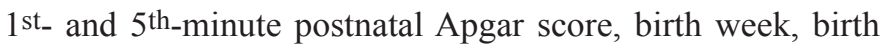
weight, and postpartum short-term medical status data were recorded. A diagnosis of pre-eclampsia was made according to the criteria of the American College of Obstetricians and Gynecologists (8). Pre-eclampsia was defined as hypertension (systolic to diastolic blood pressure $\geq 140 / 90 \mathrm{~mm} \mathrm{Hg}$ ) and proteinuria ( $>300 \mathrm{mg} /$ day in 24-hour urine collection or a protein concentration of $1 \mathrm{~g} / \mathrm{L}$ ) that started during pregnancy and ended after the birth. The $\mathrm{Hb}$ and haematocrit (Hct) values from the complete blood cell count values measured within 24 hours of birth were recorded.

\section{Imaging technique}

Placentas were clamped at a standard height on the obstetric table after the birth, put in plastic bags, and taken to the MRI unit within one hour of birth. Screening was performed in a
Philips Ingenia 1.5 T (Eindhoven, the Netherlands) machine using a SENSE Torso XL coil. Modified DIXON (mDIXON)Quant sequences were obtained. The T1 FFE mDIXON-Quant sequence parameters were TR/TE1/delta TE 5.4/0.93/0.7 ms, six echoes, flip $5 \mathrm{deg}$, BH $6 \mathrm{sec}$, slice thickness $3 \mathrm{~mm}$, matrix size $128 \times 128$, and field of view $250 \mathrm{~mm}$. Images were evaluated on the workstation (IntelliSpace Portal Philips v6.03.13200 Philips Healthcare Nederland B.V, Veenpluis 4-6, 5684 PC Best, the Netherlands). The measurements were taken on $\mathrm{T} 1 \mathrm{FFE} / \mathrm{T} 2 *$ images, which are one of the four image types (T1FFE/W, T1FFE/F, T1FFE/FF, and T1FFE/T2*) obtained in a fast single sequence. The measurements were taken using a standard ROI (region of interest) (mean 500 $\mathrm{mm}^{2}$ ) from four fields to prevent over-sensitivity to artefacts where the maximum homogeneity was obtained (Figure 1), and the mean values were obtained.

\section{Statistical analysis}

According to post hoc power analysis, using a mean T2* value parameter at alpha 0.05 level, test power achieved $83 \%$ with group sample sizes of 22 and 20. Data were expressed as mean \pm standard deviation (SD). The student's t-test and Pearson correlation test were applied to detect correlations between groups. Receiver operating characteristic curves were applied to check the diagnostic value of $\mathrm{T} 2 *$ in the estimation of pre-eclamptic placentas and the best $\mathrm{T} 2 *$ cutoff value, which represents the diagnosis of pre-eclampsia. Sensitivity and specificity were calculated based on $\mathrm{T} 2 *$ measurements. Computer software (Statistical Package for the Social Sciences Version 15.0, SPSS Inc., Chicago, IL, USA) was used for statistical analysis. A value of $p<0.05$ was accepted as statistically significant.

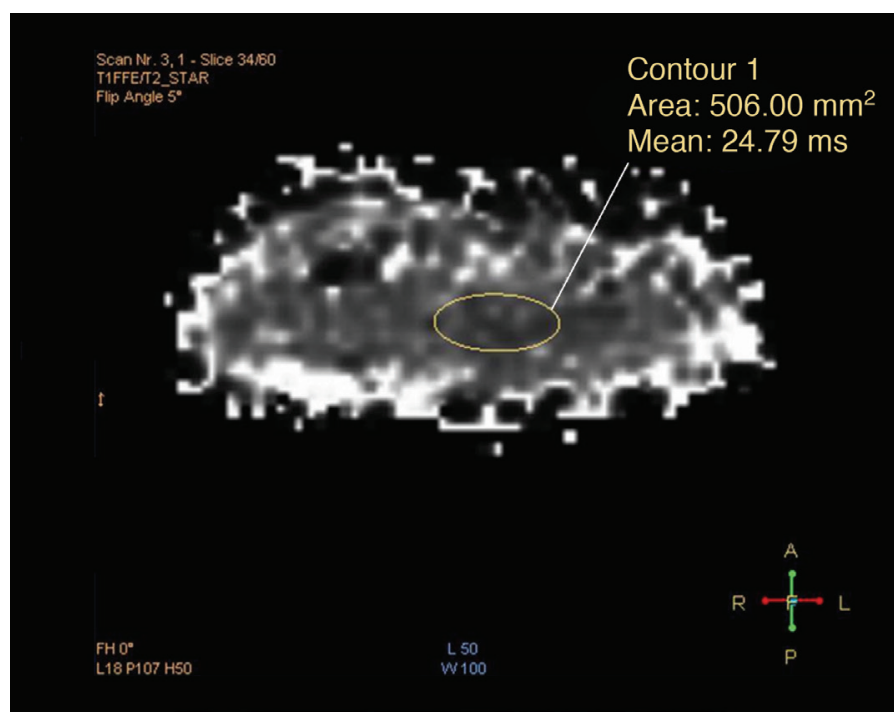

FIG. 1. T2 star image of modified DIXON-Quant sequence demonstrates placement of ROI for measurement of the placenta. 


\section{RESULTS}

Maternal ages, birth weeks, birth weights, $1^{\text {st- }}$ and $5^{\text {th-minute }}$ Apgar scores, and maternal $\mathrm{Hb}$ and Hct values are shown in Table 1 . There was no statistically significant difference between the groups in terms of mean maternal age, birth weight, 1 st. and $5^{\text {th }}$-minute Apgar scores, or maternal $\mathrm{Hb}$ and Hct levels ( $\mathrm{p}=0.093, \mathrm{p}=0.105, \mathrm{p}=0.543, \mathrm{p}=0.492, \mathrm{p}=0.128$, and $\mathrm{p}=0.128$, respectively). The mean urinary protein values of the pre-eclamptic group and the control group were $2.1 \pm 0.71$ and $0.27 \pm 0.88$, respectively. The mean systolic and diastolic tension values of the pre-eclamptic group and the control group were $162 \pm 10.05 / 98.5 \pm 7.45$ and $121.82 \pm 20.61 / 67.73 \pm 7.45$, respectively. As expected, the urinary protein and systolic and diastolic tension mean values were statistically significantly higher in the pre-eclamptic group $(\mathrm{p}<0.001$ and $\mathrm{p}<0.001$, respectively). There was no statistically significant correlation among the urinary protein, systolic and diastolic tension values, and placenta $\mathrm{T} 2 *$ values of the pre-eclamptic group $(\mathrm{p}=0.233$, $\mathrm{p}=0.730$, and $\mathrm{p}=0.148$, respectively).

A statistically significant difference was detected between the two groups in terms of $\mathrm{T} 2 *$ values $(\mathrm{p}<0.01)$ (Figure 2). While the mean $\mathrm{T}_{2} *$ value in the pre-eclamptic group was found to be $37.48 \mathrm{~ms}(\mathrm{SD} \pm 11.3)$, this value was 28.74 $(\mathrm{SD} \pm 8.08)$ in the control group. The mean birth week of the pre-eclamptic and control groups was $35 \mathrm{w} 1 \mathrm{~d} \pm 2 \mathrm{w} 4 \mathrm{~d}$ and $37 \mathrm{w}$ $6 \mathrm{~d} \pm 1 \mathrm{w} 2 \mathrm{~d}$, respectively. The birth week for the control group was significantly higher than that of the pre-eclamptic group $(\mathrm{r}=0.659, \mathrm{p}<0.001)$. There was a strong negative correlation between the $\mathrm{T} 2 *$ values of all the placentas and the birth week $(\mathrm{r}=-0.510, \mathrm{p}<0.001)$. There was a strong negative correlation between the birth week and the T2* values in the control group $(\mathrm{r}=-0.460, \mathrm{p}<0.05)$, and no correlation between birth week and

TABLE 1. Obstetric and medical features of pre-eclamptic and control groups

\begin{tabular}{lccccc}
\hline & \multicolumn{2}{c}{$\begin{array}{c}\text { Control } \\
(\mathrm{n}=22)\end{array}$} & \multicolumn{2}{c}{$\begin{array}{c}\text { Pre-eclampsia } \\
(\mathrm{n}=20)\end{array}$} & \\
\cline { 2 - 5 } & Mean & SD & Mean & SD & p \\
\hline Maternal age (year) & 31 & 5.003 & 28.3 & 5.131 & 0.093 \\
Birth week (w, d) & $37 \mathrm{w} 6 \mathrm{~d}$ & $1 \mathrm{w} 2 \mathrm{~d}$ & $35 \mathrm{w} 1 \mathrm{~d}$ & $2 \mathrm{w} 4 \mathrm{~d}$ & $<0.001^{*}$ \\
Birth weight (gr) & 3015 & 845 & 2532 & 1040 & 0.105 \\
Apgar 1 & 8.05 & 0.89 & 7.74 & 2.15 & 0.543 \\
Apgar 5 & 9.09 & 0.68 & 8.74 & 2.28 & 0.492 \\
Urine protein & 0.27 & 0.88 & 2.1 & 0.71 & $<0.001^{*}$ \\
BPS (mmHg) & 121.82 & 20.61 & 162 & 10.05 & $<0.001^{*}$ \\
BPD (mmHg) & 67.73 & 7.45 & 98.5 & 7.45 & $<0.001^{*}$ \\
Hb (g/dL) & 11.53 & 1.41 & 12.16 & 1.17 & 0.128 \\
Hct (\%) & 36.59 & 3.76 & 34.67 & 4.12 & 0.128 \\
\hline
\end{tabular}

SD: standard deviation; w: week; d: day; gr: gram; BPS: blood pressure systolic; BPD: blood pressure diastolic; Hb: haemoglobin; Hct: haematocrit

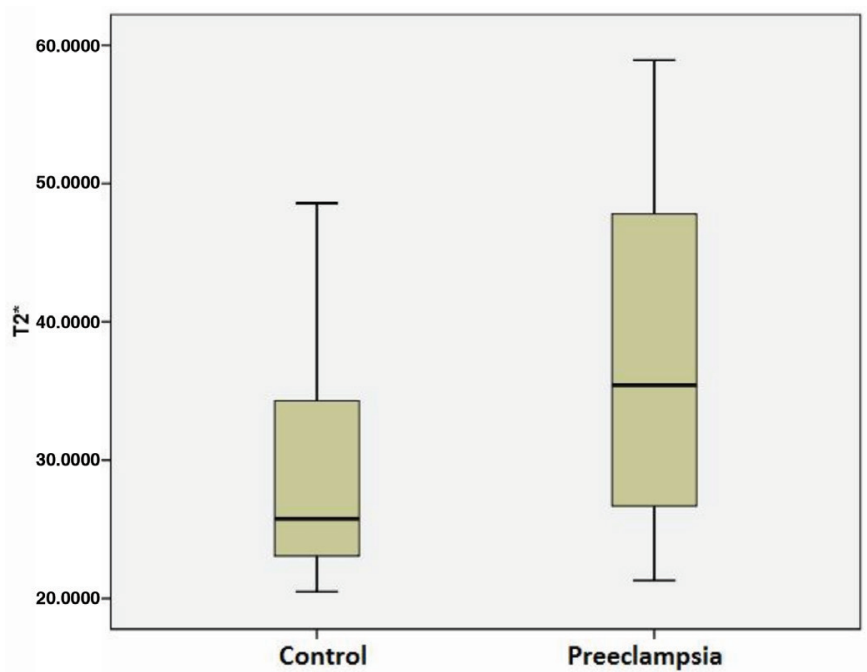

FIG. 2. Box plot showing T2 star values of pre-eclamptic and control group placentas.

$\mathrm{T} 2 *$ values in the pre-eclamptic group $(\mathrm{r}=-0.337, \mathrm{p}=0.147)$. The cutoff value for the $\mathrm{T} 2 *$ value, maximising the accuracy of diagnosis, was $28.59 \mathrm{~ms}$ (area under curve: $0.741 ; 95 \%$ confidence interval: $0.592-0.890)$; sensitivity and specificity were $70 \%$ and $63.6 \%$, respectively.

\section{DISCUSSION}

As a result of this study, which was conducted ex vivo and was the first MRI study measuring the amount of iron in the placenta, $\mathrm{T} 2 *$ values, which are the quantitative indicator of iron loading, were found to be higher in the pre-eclamptic group placentas than in the control group. Pre-eclampsia is a hypertensive dysfunction related to pregnancy-induced proteinuria, and it appears after the $20^{\text {th }}$ gestational week. This dysfunction affects $7-10 \%$ of all pregnancies and is highly related to perinatal mortality and morbidity $(9,10)$.

Although the pathophysiology of pre-eclampsia has not been clarified, genetic factors, abnormal placentation, endothelial dysfunction, and immunological interactions are described as possible pathophysiological mechanisms (11). It is known that vasoconstriction that occurs due to the increasing response to vasoactive substances, such as angiotensin II and endothelin, takes place in the pathophysiology of pre-eclampsia (12). Preeclampsia is characterised by vascular dysfunction, reduced systemic vasodilators, and increased uteroplacental resistance as a result of impaired extravillous trophoblast migration toward the uterine spiral arteries. In terms of its pathogenesis, tissue damage caused by an increase in the levels of reactive oxygen species and the altered bioavailability of nitric oxide (NO) plays an important role. Peroxynitrite, which is formed 
after superoxide quickly inactivates NO, accumulates in placental tissue and disrupts placental function. Moreover, reactive oxygen species may stimulate platelet adhesion and aggregation, causing intravascular coagulopathy. Ultimately, placental infarct increases and oxygen and nutrients decrease due to the impairment of the uteroplacental bloodstream, causing foetal growth retardation. On the other hand, antioxidants, such as catalase, superoxide dismutase, and glutathione peroxidase, clean reactive oxygen species and thus protect tissue against oxidative stress. Placental ischaemia causes oxidative stress to increase by reducing antioxidant activity, disrupting the balance between reactive oxygen types and antioxidant activity (13).

Iron, which is present in various body molecules, especially cytochrome, $\mathrm{Hb}$, and myoglobin, is stored as ferritin after it is absorbed by the mucosa of the small intestine (3). Iron loading may develop due to the malabsorption of iron, such as via hereditary hemochromatosis, a dysfunction in heme metabolism, or long-term transfusion therapy (14). While surplus iron accumulates primarily in the liver, pancreas, heart, thyroid, pituitary, and synovium, in its secondary form, it is stored in the reticuloendothelial system (kupffer cells, spleen, bone marrow, and lymph nodes). When this system is saturated, iron is stored in hepatocytes and in other parenchymal cells (15). Surplus iron may catalyse the transformation of hydrogen peroxide into free radicals, and it may cause damage to cell membranes, protein, and DNA. Iron is toxic to tissues, and it may cause hepatic, cardiac, or endocrine function disorders. In order to monitor the response to treatment, quantifying iron content is important (3). Traditionally, this measurement was taken indirectly via serum ferritin level or directly via biopsy. A weak correlation has been established between iron stored in the organs and ferritin measurements via invasive biopsy (16).

MRI is a non-invasive method that can demonstrate surplus iron loading in various organs, such as the liver, heart, spleen, and pancreas, and quantify it, and the correlations obtained in this manner are typically sound. Recently, many quantitative MRI techniques have been presented. Most are T2*-weighted, gradient echo sequence-based, and obtained using progressively longer echo times (15). There are two methods used to quantify iron loading in the liver: signal intensity ratio methods and relaxometry methods. Whereas signal intensity ratio methods measure the signal intensity and noise of the tissue $(17,18)$, relaxometry methods measure the signal intensity of the tissue for multiple echo times and thus calculate T2 or T2* values. T2 or $\mathrm{T} 2 *$ values are inversely proportional to iron concentration (19). T2* values under $18 \mathrm{~ms}$ are accepted as indicating overloading in the liver (20).
Dual-echo (2E-mDIXON), adapted from a modified DIXON (mDIXON) technique and allowing flexible echo times, is used as a fast and high-resolution fat suppression technique (2123). Six-echo mDIXON (6E-mDIXON) is perfectly correlated with forecasted hepatocyte budget and enables an estimation of T2*decays $(22,24,25)$.

As a result of normal, variant, or abnormal perfusion changes in the liver, iron loading in various forms, as well as various forms of protection from loading, have been described $(15,26)$. In one study, in the fields in which segmental signal reduction was observed in the liver via MRI, angiography, and CT portography, the decay of portal flow and the presence of abnormal iron loading were demonstrated by confirming the pathological result (26). The current study aimed to show a difference in iron amount via MRI, which would suggest that there were differing iron amounts in the placentas of the pre-eclampsia group, where there was vasoconstriction and endothelial dysfunction due to perfusion changes. With the aim of performing a quantitative evaluation, the measurement of $\mathrm{T} 2 *$ values in this study was applied using the $6 \mathrm{E}-\mathrm{mDIXON}$ sequence.

The results of this study showed that in pre-eclamptic placentas with perfusion dysfunction in their pathophysiology, the amount of iron was found to be less than that of the control group. While iron amounts differed between the groups, no difference was detected with regard to maternal $\mathrm{Hb}$ and Hct values. There was a difference between the birth weeks of the two groups, and in the in-group correlation analysis, there was a negative correlation between the $\mathrm{T} 2 *$ value and birth week of the control group (i.e., as the weeks progressed, the amount of iron in the placenta increased). No significant difference was determined in $\mathrm{T} 2 *$ values as birth weeks progressed in the pre-eclamptic group. This may be because of a reduced blood flow to the placenta due to endothelial dysfunction and vasoconstriction, which may have caused less iron loading in the placenta. Based on the results of this study, there is a need for further in vivo MRI studies in which the amount of iron in the placenta is measured. With the help of that sequence, which could be performed in a short time, and measurement of the iron amount, a non-invasive screening method could be used in the early diagnosis and follow-up of pre-eclampsia.

There have been various MRI studies of pre-eclamptic placentas $(27,28)$. However, there are no studies that measure the amount of iron in the placenta via MRI. Thus, the values obtained in this study could not be compared with any in the literature.

This study has certain limitations. The control group was formed randomly. As expected, a difference was detected in the birth week for the pre-eclampsia and control groups. There was no histopathological evaluation of the placentas. Concurrent 
histopathological studies with MRI may reveal the relationship between structural placenta changes and the MRI findings. It is hoped that this article will encourage future in vivo MRI studies with larger patient groups and studies supported by histopathological correlation.

Iron loading is relatively common and has various causes. Information based on screening findings may provide useful data during the process of diagnosis and treatment. MRI is the best non-invasive screening method for quantitative measurement of the amount of iron. At the end of the current study, the iron amount in pre-eclamptic placentas was found to be lower than that of the control group. This could have developed secondarily to vasoconstriction and endothelial dysfunction. Further studies of larger patient groups and with histopathological confirmation are needed.

Financial disclosure: The financial support of this work has been covered by the authors.

Conflict of interest: No conflict of interest was declared by the authors.

\section{REFERENCES}

1. Roberts JM, Taylor RN, Musci TJ, Rodgers GM, Hubel CA, McLaughlin MK. Preeclampsia: an endothelial cell disorder. Am J Obstet Gynecol $1989 ; 161: 1200-4$

2. Lam C, Lim KH, Karumanchi SA. Circulating angiogenic factors in the pathogenesis and prediction of preeclampsia. Hypertension 2005;46:107785.

3. Andrews NC. Disorders of iron metabolism. N Engl J Med 1999;341:198695.

4. Alústiza JM, Artetxe J, Castiella A, Agirre C, Emparanza JI, Otazua P, et al. MR quantification of hepatic iron concentration. Radiology 2004;230:47984.

5. Gomori JM, Horev G, Tamary H, Zandback J, Kornreich L, Zaizov R, et al. Hepatic iron overload: quantitative MR imaging. Radiology 1991;179:3679.

6. Westphalen AC, Qayyum A, Yeh BM, Merriman RB, Lee JA, Lamba A, et al. Liver fat: effect of hepatic iron deposition on evaluation with opposedphase MR imaging. Radiology 2007;242:450-5.

7. Li TQ, Aisen AM, Hindmarsh T. Assessment of hepatic iron content using magnetic resonance imaging. Acta Radiol 2004;45:119-29.

8. ACOG Committee on Practice Bulletins Obstetrics. ACOG practice bulletin. Diagnosis and management of preeclampsia and eclampsia. Number 33, January 2002. Obstet Gynecol 2002;99:159-67.

9. Chappell LC, Enye S, Seed P, Briley AL, Poston L, Shennan AH. Adverse perinatal outcomes and risk factors for preeclampsia in women with chronic hypertension: a prospective study. Hypertension 2008;51:1002-9.
10. No authors listed. National High Blood Pressure Education Program Working Group Report on High Blood Pressure in Pregnancy. Am J Obstet Gynecol 1990;163:1691-712.

11. Turgal M, Beksac MS. Pathophysiology of Preeclampsia. Turkiye Klinikleri J Gynecol Obst-Special Topics 2012;5:13-6.

12. Brown MA, Wang J, Whitworth JA. The renin-angiotensin-aldosterone system in pre-eclampsia. Clin Exp Hypertens 1997;19:713-26.

13. Matsubara K, Higaki T, Matsubara Y, Nawa A. Nitric oxide and reactive oxygen species in the pathogenesis of preeclampsia. Int $\mathrm{J}$ Mol Sci 2015;16:4600-14.

14. Wood JC, Ghugre N. Magnetic resonance imaging assessment of excess iron in thalassemia, sickle cell disease and other iron overload diseases. Hemoglobin 2008;32:85-96.

15. İdilman İS, Akata D, Özmen MN, Karçaaltıncaba M. Different forms of iron accumulation in the liver on MRI. Diagn Interv Radiol 2016;22:22-8.

16. Anderson LJ, Holden S, Davis B, Prescott E, Charrier CC, Bunce NH, et al. Cardiovascular T2-star (T2*) MR for the early diagnosis of myocardial iron overload. Eur Heart J 2001;22:2171-9.

17. Bonkovsky HL, Rubin RB, Cable EE, Davidoff A, Rijcken TH, Stark DD. Hepatic iron concentration: noninvasive estimation by means of MR imaging techniques. Radiology 1999;212:227-34.

18. Gandon Y, Guyader D, Heautot JF, Reda MI, Yaouanq J, Buhé T, et al. Hemochromatosis: diagnosis and quantification of liver iron with gradientecho MR imaging. Radiology 1994;193:533-8.

19. Alexopoulou E, Stripeli F, Baras P, Seimenis I, Kattamis A, Ladis V, et al. R2 relaxometry with MRI for the quantification of tissue iron overload in beta-thalassemic patients. J Magn Reson Imaging 2006;23:163-70.

20. Westwood M, Anderson LJ, Firmin DN, Gatehouse PD, Charrier CC, Wonke B, et al. A single breath-hold multiecho $\mathrm{T} 2 *$ cardiovascular magnetic resonance technique for diagnosis of myocardial iron overload. J Magn Reson Imaging 2003;18:33-9.

21. Berglund J, Ahlström H, Johansson L, Kullberg J. Two-point dixon method with flexible echo times, Magn Reson Med 2011;65:994-1004.

22. Eggers H, Brendel B, Duijndam A, Herigault G. Dual-echo Dixon imaging with flexible choice of echo times. Magn Reson Med 2011;65:96-107.

23. Dixon WT. Simple proton spectroscopic imaging. Radiology 1984;153:18994.

24. Kukuk GM, Hittatiya K, Sprinkart AM, Eggers H, Gieseke J, Block W, et al. Comparison between modified Dixon MRI techniques, MR spectroscopic relaxometry, and different histologic quantification methods in the assessment of hepatic steatosis. Eur Radiol 2015;25:2869-79.

25. Yu H, Shimakawa A, McKenzie CA, Brodsky E, Brittain JH, Reeder SB. Multiecho water-fat separation and simultaneous R2* estimation with multifrequency fat spectrum modeling. Magn Reson Med 2008;60:112234.

26. Kadoya M, Matsui O, Kitagawa K, Kawamori Y, Yoshikawa J, Gabata T, et al. Segmental iron deposition in the liver due to decreased intrahepatic portal perfusion: findings at MR imaging. Radiology 1994;193:671-6.

27. Austdal M, Thomsen LC, Tangerås LH, Skei B, Mathew S, Bjørge L, et al. Metabolic profiles of placenta in preeclampsia using HR-MAS MRS metabolomics. Placenta 2015;36:1455-62.

28. Huen I, Morris DM, Wright C, Parker GJ, Sibley CP, Johnstone ED, et al. $\mathrm{R} 1$ and $\mathrm{R} 2 *$ changes in the human placenta in response to maternal oxygen challenge. Magn Reson Med 2013;70:1427-33. 